\title{
Correction to: Weighted feature-task-aware regularization learner for multitask learning
}

Wei Xue ${ }^{1}$ (D)

Published online: 1 June 2019

(c) Springer-Verlag London Ltd., part of Springer Nature 2019

\section{Correction to: Pattern Analysis and Applications https://doi.org/10.1007/s10044-019-00781-8}

\begin{abstract}
: these sentences "share knowledge between tasks and improve the collective performance", "how to enhance the sparsity of the learned variables is not taken into consideration" and "Finally, we conduct experiments to compare the resulting approach with some related methods used for multitask learning, which illustrates the efficiency of the proposed method." should be deleted.
\end{abstract}

1 Introduction: page 2, the ninth line on the left side, the sentence "The major contributions of our work are four three folds:" should be deleted.

3.1 Problem setup: page 3, the first line on the left side, "and", "a" and "same" should be deleted. "the Q decision" in the thirteenth line should be deleted.

3.2 Model formulation: page 3, "can be" in the first line on the left side, "minimization" in the tenth line, "additional" in the thirteenth line, the sentence " $\mu>0$ controls the sparsity of the solutions on task level, and $\sigma_{j}>0$ controls the sparsity of the solutions on feature level" in the sixteenth line, "should" and "while keeping" in the twentieth line on the right side should be deleted.

4.1 Learning algorithm: page 4, "at each" in the third line and "a positive parameter" in the fifth line on the right side should be deleted. Page 5, "minimization" in the fourth line on the left side should be deleted.

4.2 Theoretical analysis: page 5, "the regret is defined by" in the second line, "where" in the fifth line, "methods" in the twentieth line, and "the RDA-WIFAR method

The original article can be found online at https://doi.org/10.1007/ s10044-019-00781-8.

Wei Xue

cswxue@ahut.edu.cn

1 School of Computer Science and Technology, Anhui University of Technology, Maanshan 243032, China converges" in the twenty-first line on the right side should be deleted.

5 Performance evaluation: page 6, "all adopt" in the first line and "regularized dual averaging method for" in the third line on the right side should be deleted. Page 7, "regularized dual averaging method for" in the first and third lines, and "regularized dual averaging" in the seventh line on the left side should be deleted.

6 Conclusion: "our" in the eighth line should be deleted.

The author regrets these errors and apologizes for any inconvenience caused.

Publisher's Note Springer Nature remains neutral with regard to jurisdictional claims in published maps and institutional affiliations. 